\title{
Analysis and Calculation of the Best Center of Mass for Supercavitating Projectile
}

\author{
Ping Wei $\left(D,{ }^{1}\right.$ Shoufa Wang, ${ }^{1}$ Wenrong Yan, ${ }^{1,2}$ and Xin Yu ${ }^{1}$ \\ ${ }^{1}$ College of Weaponry Engineering, Naval University of Engineering, Wuhan 430033, China \\ ${ }^{2} X i$ 'an Modern Chemistry Research Institute, Xi'an 710065, China \\ Correspondence should be addressed to Ping Wei; pingwei501@126.com
}

Received 21 December 2021; Accepted 19 January 2022; Published 23 February 2022

Academic Editor: Guolong Shi

Copyright (c) 2022 Ping Wei et al. This is an open access article distributed under the Creative Commons Attribution License, which permits unrestricted use, distribution, and reproduction in any medium, provided the original work is properly cited.

Currently, the supercavitating projectiles mostly rely on experience or experimental results to test the shape of the projectile; however, the cost of the experiment is relatively high, and there is no specific criterion to judge whether the underwater projectile is stable. To solve the aforementioned problems, we study the motion stability and establish motion equations for supercavitating projectiles. Through theoretical analysis and simulation calculations, the optimal center of mass position is designed to optimize the motion performance of underwater supercavitating projectiles. We think this work can provide theoretical support for the optimal design of underwater supercavitating projectiles.

\section{Introduction}

When the vehicle is moving at high speed underwater, supercavitation is formed on the surface of the vehicle. Supercavitation can significantly reduce the viscous resistance of the vehicle when it is moving underwater and greatly increase the speed and range of the navigation [1]. For high-speed vehicles, small changes in the position of the center of mass, the structure of the supercavity, and other factors will seriously affect the navigation speed and range.

At present, there are a lot of researches on the theoretical analysis of supercavitating projectiles through mathematical models for reference. For the study of high-speed supercavitating projectiles, starting from the perspective of dynamics, a motion equation is established for the projectile in the supercavitation cavity to study the impact of projectile quality and supercavity structure on the flight performance of supercavitating projectiles [2]. The increase in the mass of the projectile can reduce the deviation of the projectile trajectory, and the increase in the diameter of the supercavity will increase the deviation of the trajectory. Using a water tunnel, the literature [3] studied the hydrodynamic problem of a cylindrical vehicle tail when sliding inside the cavity.
Cylindrical voyages interact with each other in the supercavity, and this impact also affects the supercavity. The impact is more pronounced during longitudinal movement. By analyzing the fluid dynamics of the supercavitating projectile, the nonlinear dynamic equation of motion is obtained. According to the stability equation, the control parameters that affect the stability of the supercavitating projectile are obtained [4-6]. The movement process of a projectile after entering the water is usually divided into an initial stage and a tail shot stage. In the tail shot stage, the tip force received by the high-speed supercavitating projectile is generally along the opposite direction of the projectile's pointing motion [7-9]. Affected by the projectile entering water, the high-speed supercavitating projectile rotates in the cavity while advancing, which is this rotation that causes the tail of the projectile to collide with the cavity wall [10]. As shown in the literature [11], as the projectile beats the water wall, the force of the water wall on the projectile tail gradually decreases, and finally, the supercavitation collapses. The literature [12] obtained through theoretical analysis of the force of the projectile: when the projectile has the same shape and other conditions such as the length of the projectile, the forward movement of the center of mass is beneficial to the linear movement of the projectile and is beneficial to 
keep stability of movement of the projectile underwater. The collision of the projectile with the cavity wall can reduce the projectile energy consumed by the tail of the projectile, and the collision is conducive to the linear movement of the projectile [13]. The selection of denser materials for the warhead is conducive to the forward movement of the center of mass and the linear motion of the supercavitating projectile. When the shape of the projectile remains unchanged, adding high-density materials in the projectile body or adding a stabilizer tube to the tail of the projectile can improve the stability of the supercavitating projectile, which provides a basis for the projectile design. Meng et al. [14] studied the motion characteristics of underwater supercavitating projectiles through kinematics and dynamics analysis and mathematical modeling and found that the angular velocity of the projectile changes periodically. The decrease in the size of the supercavitation will cause the tail shot amplitude decreases; the smaller the projectile's moment of inertia, the more stable the tail shot changes, the launch speed, and the launch depth. The larger initial angular velocity makes the tail angular velocity of the projectile decay faster. Zhang and Hou [15] analyzed the stability of underwater projectiles and found that the shape design of underwater projectiles has a great influence on the stability of motion. The larger the aspect ratio of the projectile shape, the more stable the movement of the underwater projectile. Zhao et al. [16] studied the influence of the position of the center of mass on the motion of the tail shot and obtained the effect of the tail shot motion on the symmetry of the supercavitation, and the supercavity surface wall at the collision point develops in the direction of the supercavitating projectile rotation. During the movement of the supercavitating projectile, the angular velocity, angular acceleration, and restoring force of the tail shot all change periodically. Zheng [17], Qian [18], and Huang [19] all conducted simulation studies on projectiles entering the water. The viscous resistance of supercavitating projectiles under water is proportional to the square of the sailing speed. When entering the water at the same inclination angle, the greater the water entering speed, the easier it is to produce obvious cavitation, and the force on the surface of the projectile reaches the peak at the moment of entering the water. Shi et al. [20] carried out resistance analysis and motion stability analysis of continuous-launched projectiles through fluent software. The flow field generated by the first supercavitating projectile is helpful to reduce the pressure on the head of the projectile. When the bubble projectile enters the supercavitation of the front bullet, the head of the projectile will hardly be affected by viscous resistance, causing the projectile to rear-end and split the supercavitation. Yi and Xiong [21] studied the resistance characteristics of natural supercavitation flow of high-speed vehicles and found that the drag coefficient of high-speed vehicles decreases with the increase of speed. The increase of the slenderness ratio of the vehicle is beneficial to reduce the drag coefficient of small highspeed craft, and the drag reduction rate of high-speed craft reaches more than 95\%. Zhang et al. [22] studied the influencing factors of the impact of the tail shot and concluded that the increase of the mass increases the force of the single tail shot, but as the center of mass moves backward, the number of tail shots will be reduced; that is, the single tail shot will be increased.

To sum up, for most underwater projectiles, the position of the center of mass is set according to experience; however, there is no corresponding theory to solve such problem. Based on the analysis of the movement characteristics of the projectile in water, star $\mathrm{ccm}+$ fluid simulation software is used for ballistic simulation, and the best position of the center of mass is found by constantly changing the position of the center of mass. We think this work can provide theoretical support for the design of supercavitating projectiles.

\section{Mathematical Model}

2.1. Equation of Motion. The projectile rotates continuously during launch and movement in the water. If the center of mass is not properly positioned, the projectile will roll, which will severely shorten the range of the projectile. The projectile must satisfy the following equations of motion:

$$
\begin{aligned}
M \dot{U}_{\mathrm{cm}} & =\sum F_{X 1}, \\
M \dot{W}_{\mathrm{cm}} & =\sum F_{Z 1}, \\
I \dot{Q} & =\sum M_{Y 1} .
\end{aligned}
$$

Among them, $M$ is the mass of the projectile, $U_{\mathrm{cm}}$ and $W_{\mathrm{cm}}$ are the velocity of the projectile's center of mass along the coordinate system axis $X 1$ and $Z 1, I$ is the moment of inertia around the $Y 1$ axis and passing through the center of mass, $Q^{\bullet}$ is the angular acceleration, and $M_{Y 1}$ is the resultant moment [1].

In the initial stage, the projectile mainly considers the front-end resistance $F_{D}$ of the projectile and its own gravity. In the tail shot stage, the collision between the projectile and the bubble wall needs to be considered. The motion equation of the projectile is relatively complicated, and by simplifying the equation, we can get the following motion equations:

$$
\begin{aligned}
\dot{Q} & =\frac{\mathrm{mg}}{I}\left(L-x_{\mathrm{cm}}\right), \\
\sum M_{Y 1} & =\operatorname{mg}\left(L-x_{\mathrm{cm}}\right), \\
\dot{Q} & =-\left\{M_{2}\left[W^{2}\left(L l_{k}\right)+2 W Q\left(L^{2} l_{k}\right)\right]-\operatorname{mg}\left(L-x_{\mathrm{cm}}\right)\right\}, \\
\sum M_{Y 1} & =M_{I}+\operatorname{mg} \cos \theta\left(L-x_{\mathrm{cm}}\right) .
\end{aligned}
$$

In the formula, $x_{\mathrm{cm}}$ is a variable, and the total moment $\sum M_{Y 1} \geq 0$ always holds. In the initial stage, when $x_{\mathrm{cm}}$ becomes larger, the projectile is easier to move along a straight line. In the tail shot stage, $x_{\mathrm{cm}}$ becomes larger, $\sum$ $M_{Y 1}$ becomes smaller, and $\dot{Q}$ becomes larger. It is conjectured that there is a critical mass center $x_{\mathrm{cm}}$ value. If $x_{\mathrm{cm}}$ is less than the critical value, the underwater projectile will be unstable. If the value is greater than this critical value, the 
underwater projectile can maintain the stability of the movement.

2.2. Governing Equation. For turbulent flow, the NavierStokes equation is always applicable [23]. The following is the mass conservation equation:

$$
\frac{\partial \rho}{\partial t}+\frac{\partial}{x_{i}}\left(\rho u_{i}\right)=0
$$

where $\rho$ is fluid density and $u_{i}$ is the component of the fluid velocity along the $i$ direction.

The following is the momentum conservation equation:

$$
\frac{\partial \rho}{\partial t}\left(\rho u_{i}\right)+\frac{\partial}{\partial x_{j}}\left(\rho u_{i} u_{j}\right)=-\frac{\partial p}{\partial x_{i}}+\frac{\partial \tau_{i j}}{\partial x_{j}}+\rho g_{i}+F_{i}
$$

The following is the energy conservation equation:

$$
\frac{\partial}{\partial t}(\rho h)+\frac{\partial}{\partial x_{i}}\left(\rho u_{i} h\right)=\frac{\partial}{\partial x_{i}}\left(k+k_{i}\right) \frac{\partial T}{\partial x_{i}}+S_{h}
$$

where $p$ is the static pressure, $\tau_{i j}$ is the stress vector, $g_{i}$ is the gravity component in the $i$ direction, $F_{i}$ is other energy terms caused by resistance and energy, $h$ is the entropy, $k_{i}$ is conductivity of the turbulence transfer, $S_{h}$ is the defined volume source, and $k$ is the molecular conductivity.

\subsection{Basic Model of Star ccm+}

2.3.1. The VOF Model. The research content of this study involves the process of projectile entering water and the process of forming supercavity in water, so it involves threephase flow, namely, air phase, water phase, and water vapor phase. In addition, the DFBI 6-degree-of-freedom motion is used in the simulation to simulate the whole process of the projectile movement, so the VOF multiphase model and the VOF wave model are used.

The VOF multiphase model is a simple multiphase model. It is suitable for simulation processing where there are clear and obvious interfaces between multiphases and no mutual dissolution problem occurs between phases. It is often used in gas-liquid flow and liquid-liquid flow problems, and it focuses on the shape of the phase interface. It is worth noting that the VOF model is not suitable for the case when the scale of the phase interface is much smaller than the mesh size, because the calculation accuracy is very low at this time. The VOF multiphase model is a uniform and homogeneous multiphase flow model; that is, all phases share the same velocity field, and there are gas phase, liquid phase, and vapor phase in the calculation results, but in the actual solution process, the three phases share the same. The velocity field is when solving the equation, on the same grid, the gas phase, the liquid phase, and the vapor phase share a set of momentum equations to solve, which simplifies the calculation.

To simulate multiple insoluble fluid flows on a numerical grid, the VOF wave model is used to simulate surface gravity waves at the interface between light and heavy fluids; this model is often used in marine applications with a 6DOF motion model. This study involves the process of projectile entering water and the process of forming cavitation in water, so it involves three-phase flow, namely, air phase, water phase, and water vapor phase. And the simulation uses DFBI 6-degree-of-freedom motion to simulate the whole process of projectile motion, so the VOF multiphase model and VOF wave model are used.

2.3.2. The Turbulence Model. The governing equations of the $K$-epsilon turbulence model are listed as follows.

The following is the $K$ equation:

$$
\rho \frac{d k}{d t}=\frac{\partial}{\partial x_{i}}\left[\left(\mu_{l}+\frac{\mu_{t}}{\sigma_{k}}\right) \frac{\partial k}{\partial x_{i}}\right]+G_{k}+G_{b}-\rho \varepsilon
$$

The following is the $\varepsilon$ equation:

$$
\rho \frac{d \varepsilon}{d t}=\frac{\partial}{\partial x_{i}}\left[\left(\mu+\frac{\mu_{t}}{\sigma_{\varepsilon}}\right) \frac{\partial \varepsilon}{\partial x_{i}}\right]+C_{1 \varepsilon} \frac{\varepsilon}{k}\left(G_{k}+C_{3 \varepsilon} G_{b}\right)-C_{2 \varepsilon} \rho \frac{\varepsilon^{2}}{k} .
$$

In the above equations, $\mu_{l}$ is the laminar viscosity coefficient, $\mu_{t}$ is the turbulent viscosity coefficient, $\mu_{t}=\rho C_{\mu} k^{2} / \varepsilon$ ( $C_{\mu}$ is the turbulence constant), $G_{K}$ is the turbulent kinetic energy generated by the laminar velocity gradient, $G_{b}$ is the turbulent kinetic energy generated by buoyancy, and $\mathrm{C}_{1 \varepsilon}$, $C_{2 \varepsilon}, C_{3 \varepsilon}, \sigma_{\varepsilon}$, and $\sigma_{k}$ are empirical constants. According to the turbulence experiment, the empirical constants are $\mathrm{C}_{1 \varepsilon}=1.44, C_{2 \varepsilon}=1.92, C_{3 \varepsilon}=0.09, \sigma_{\mathrm{k}}=1.0$, and $\sigma_{\varepsilon}=1.3$.

2.3.3. The Supercavity Model. The complete Rayleigh-Plesset supercavity model includes the effects of the acceleration of bubble growth rate, the effects of viscosity, and surface tension. The bubble growth rate $v$ is determined using the Rayleigh-Plesset equation.

$$
R \frac{d_{v}}{d_{t}}+\frac{3}{2} v^{2}=\frac{p_{\mathrm{sat}}-p}{p_{l}}-\frac{2 \sigma}{\rho_{l} R}-4 \frac{\mu_{l}}{\rho_{l} R} v
$$

Among them, $p_{\text {sat }}$ is the saturation pressure at a given temperature, $p$ is the partial pressure in the surrounding liquid, $\sigma$ is the surface tension, and $\rho_{1}$ is the liquid density.

The growth rate of supercavity bubbles uses an inertial control growth model.

$$
v_{r}^{2}=\frac{2}{3}\left(\frac{p_{\mathrm{sat}}-p}{\rho_{l}}\right)
$$

where $v_{r}$ is the growth rate of supercavity bubbles.

2.4. Boundary Condition Setting. The inlet boundary condition of the velocity is applicable with incompressible flow and subsonic compressible flow. At the boundary, star $\mathrm{ccm}$ + will impose or calculate the velocity $v$, the static pressure $P_{s}$, and the temperature $T_{s}$ according to the flow conditions.

The pressure outlet boundary is the outflow condition of the applied working pressure, which can be regarded as the 


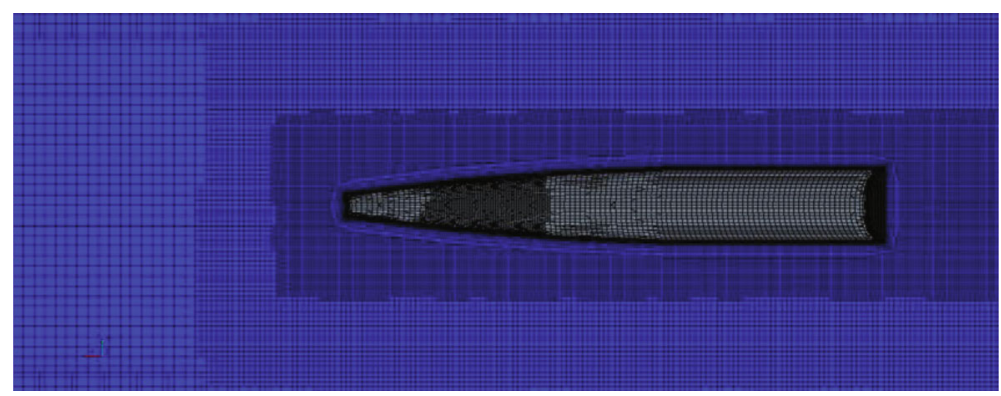

Figure 1: The computational model.

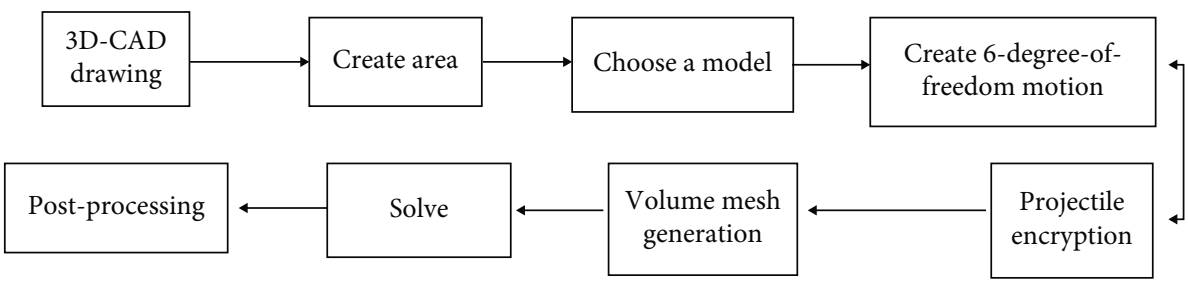

FIgURE 2: The modeling process.

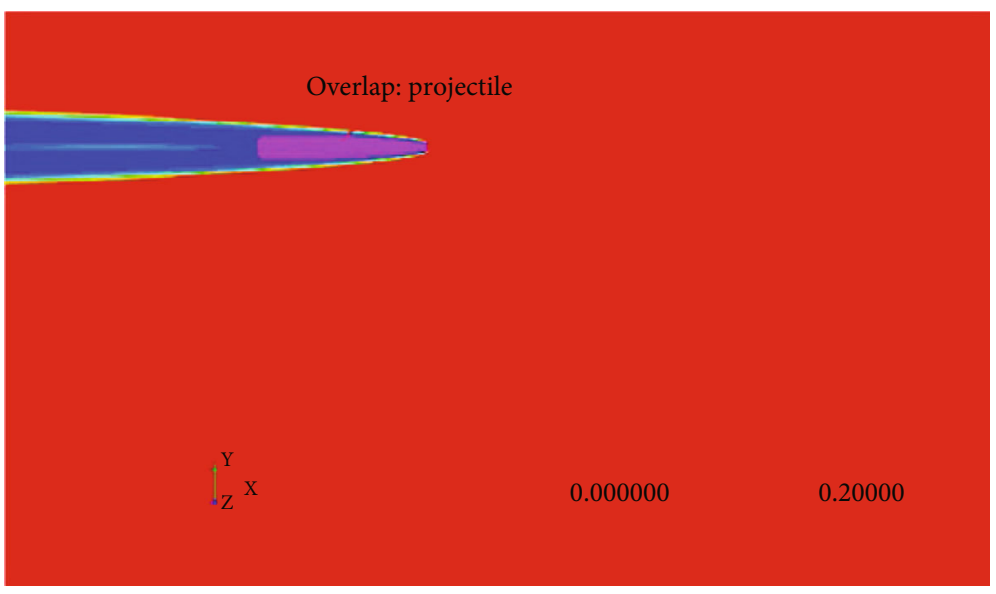

FIGURE 3: Intermediate movement process diagram of supercavitating projectile.

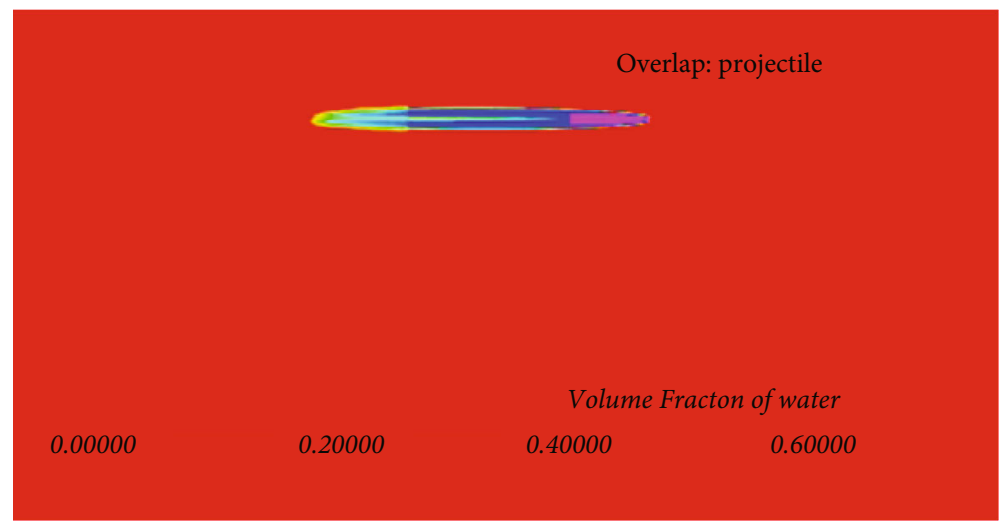

FIGURE 4: Supercavitating projectile at $200 \mathrm{~m} / \mathrm{s}$.

static pressure of the fluid when the fluid enters into the environment. Under normal outflow conditions, the boundary surface values of all other variables such as velocity or temperature can be extrapolated from inside the solution domain. For the pressure outlet boundary, star $\mathrm{ccm}+$ will be applied according to the flow situation or calculated at 


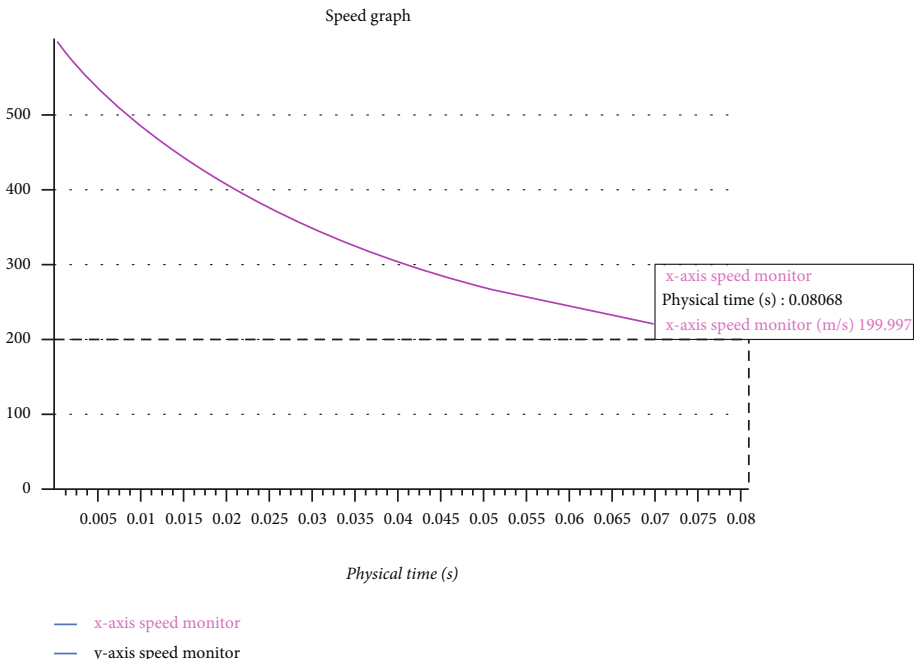

Figure 5: Graph of velocity change of supercavitating projectile.

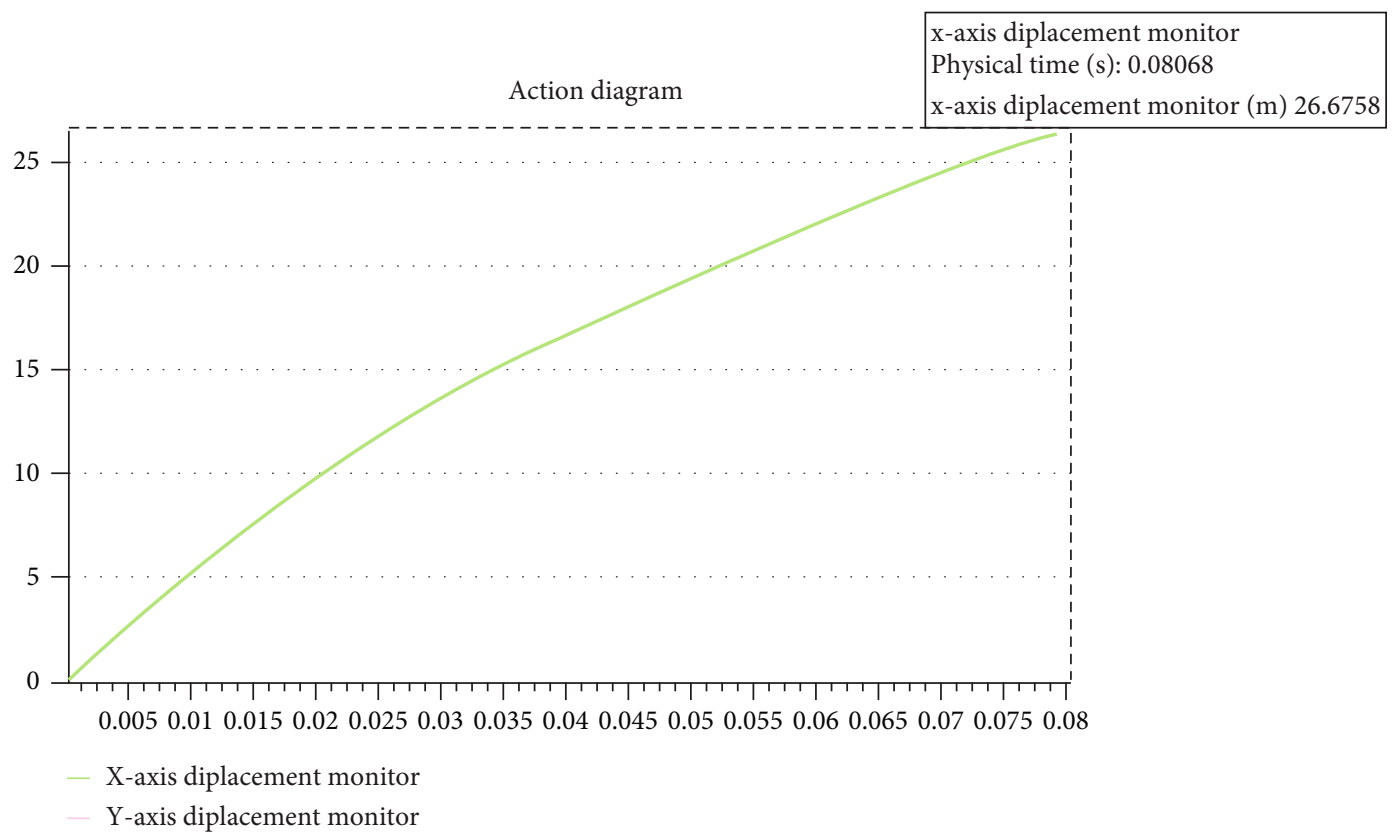

FIGURE 6: Graph of the displacement of supercavitating projectile.

the boundary surface: the velocity $v$, the static pressure $P_{s}$, and the temperature $T_{s}$ according to the flow conditions.

2.5. Calculation Model. The projectile can be regarded as a rigid body, and its total length is $L$. By changing the length of the projectile, the shape of the projectile, and the position of the center of mass and comparing the range when decelerating to $200 \mathrm{~m} / \mathrm{s}$, the influence of the change of the shape on the drag reduction performance can be judged.

The projectile adopts three truncated cones with a flat head, and the cone angle of each truncated cone gradually becomes smaller and the width of the truncated cone gradually becomes larger from the back of the head. The projectile in this simulation experiment is on the left side of the computational domain with a length of $40 \mathrm{~m}$, a width of $1.5 \mathrm{~m}$, and a height of $3 \mathrm{~m}$. The grid of the computational domain
TABLE 1: Simulation results.

\begin{tabular}{lcc}
\hline$x_{\mathrm{cm}}(\mathrm{L})$ & $\begin{array}{c}\text { Time } t / s \text { when the speed } \\
\text { drops to about } 200 \mathrm{~m} / \mathrm{s}\end{array}$ & Displacement $x(\mathrm{~m})$ \\
\hline 0.05 & 0.8068 & 26.6758 \\
0.25 & 0.8068 & 26.6706 \\
0.5 & 0.8068 & 26.6673 \\
0.6 & $/$ & 3.48 \\
\hline
\end{tabular}

Note: when the position of the center of mass is $0.6 \mathrm{~L}$ from the head of the projectile, the underwater projectile will roll during the movement. At $0.01 \mathrm{~s}$, the speed is almost zero and the displacement is $3.48 \mathrm{~m}$.

is divided into a background grid and an overlapping grid. The computational model is shown in Figure 1. The projectile body is then encrypted in the overlapping grid. In the 


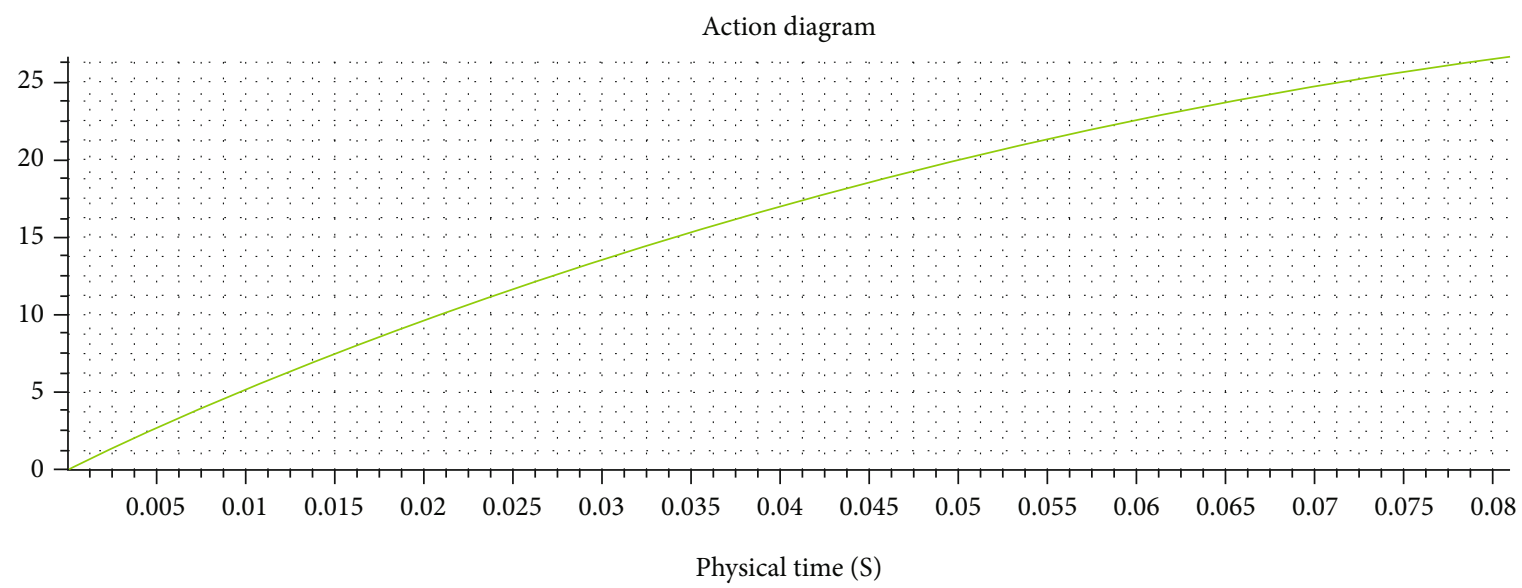

_ $\mathrm{x}$-axis displacement monitor

_ $\mathrm{y}$-axis displacement monitor

FIGURE 7: Graph of the displacement of supercavitating projectile when centroid position equals $0.55 \mathrm{~L}$.

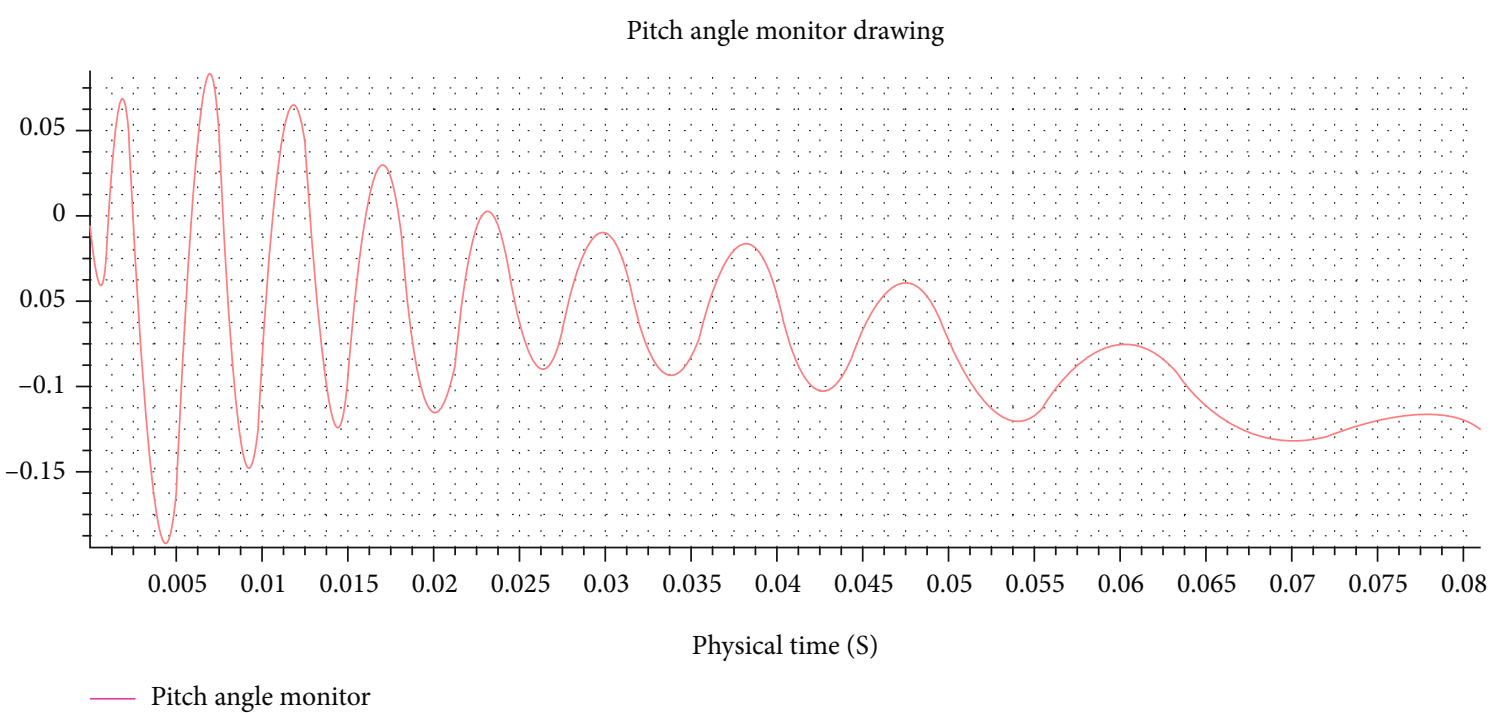

Figure 8: Graph of the elevation angle change of supercavitating projectile when centroid position equals $0.55 \mathrm{~L}$.

calculation process, the projectile body interacts with the overlapping area, and the overlapping grid interacts with the background grid. The modeling process is shown in Figure 2.

\section{Numerical Simulation Study}

The center of mass position is $0.05 \mathrm{~L}, 0.25 \mathrm{~L}, 0.5 \mathrm{~L}, 0.55 \mathrm{~L}$, $0.57 \mathrm{~L}$, and $0.6 \mathrm{~L}$ from the warhead. The middle motion process graph, the velocity graph, and the displacement graph at $200 \mathrm{~m} / \mathrm{s}$ are used to judge the projectile movement performance under the water. From the above process, the best center of mass position could be found.

3.1. The Influence of the Position of the Center of Mass on the Movement Performance of Underwater Projectiles. Take the simulation result when the center of mass is $0.05 \mathrm{~L}$ away from the warhead as an example.
It can be seen from Figures 3-6 that when the center of mass is $0.05 \mathrm{~L}$ from the projectile head, at $0.8068 \mathrm{~s}$, the speed drops to about $200 \mathrm{~m} / \mathrm{s}$ and the displacement is $26.6758 \mathrm{~m}$. Also, the simulation results are listed in Table 1.

3.2. The Effect of the Center of Mass Position on the Range. The three simulation results of the center of mass of $0.05 \mathrm{~L}$, $0.25 \mathrm{~L}$, and $0.5 \mathrm{~L}$ from the head of the projectile show that under the premise of stable underwater projectile movement, the front of the center of mass has little effect on the range of the underwater projectile.

There are some reasons to explain the inconsistency between the simulation results and the theoretical results. The center of mass reduces the single energy consumption of the underwater projectile's tail shot motion. As energy consumption is reduced, the attenuation of angular velocity and angular acceleration becomes slower, the frequency of the up and down vibration of the projectile in the cavity 


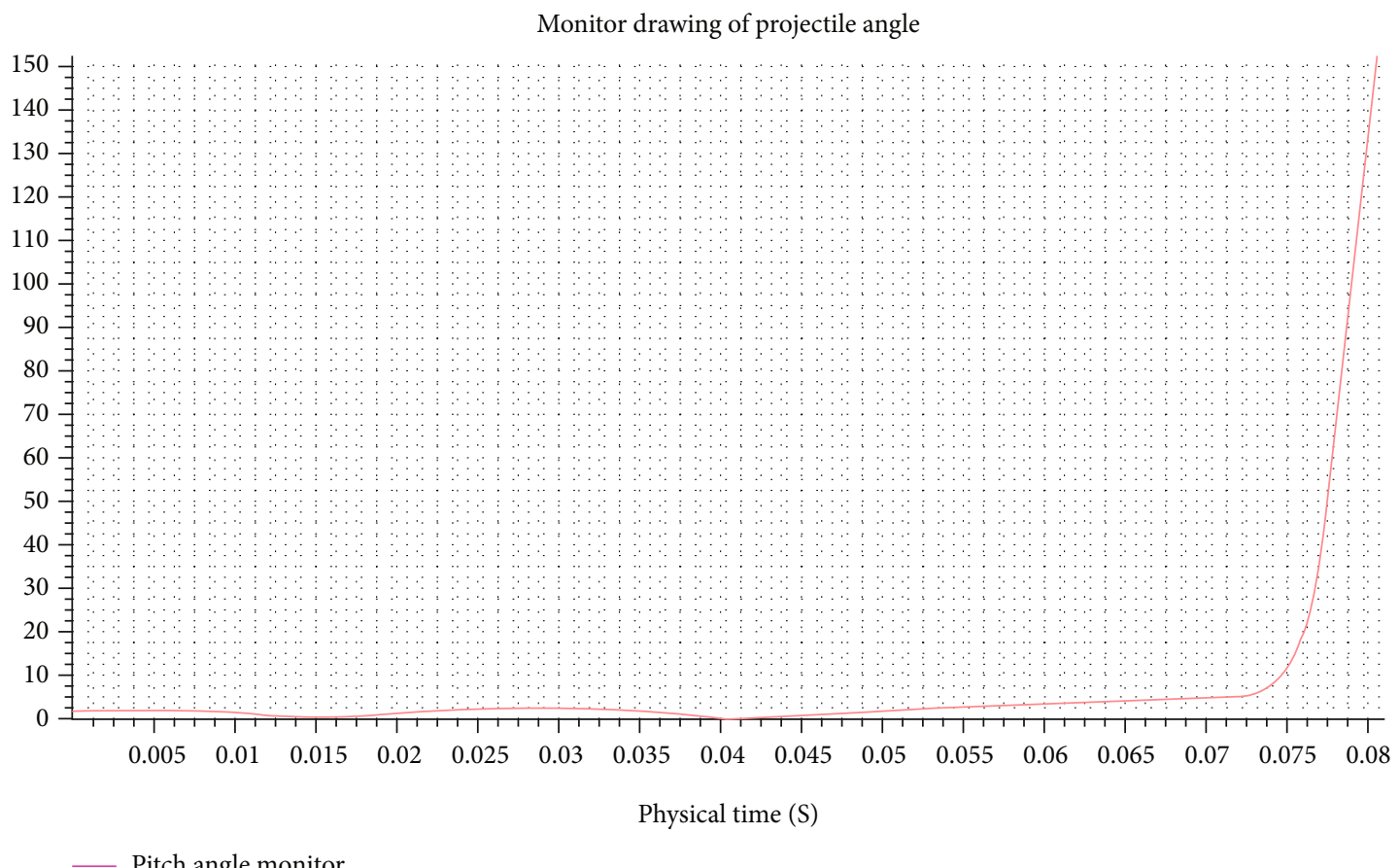

Figure 9: Graph of the elevation angle change of supercavitating projectile when centroid position equals $0.56 \mathrm{~L}$.

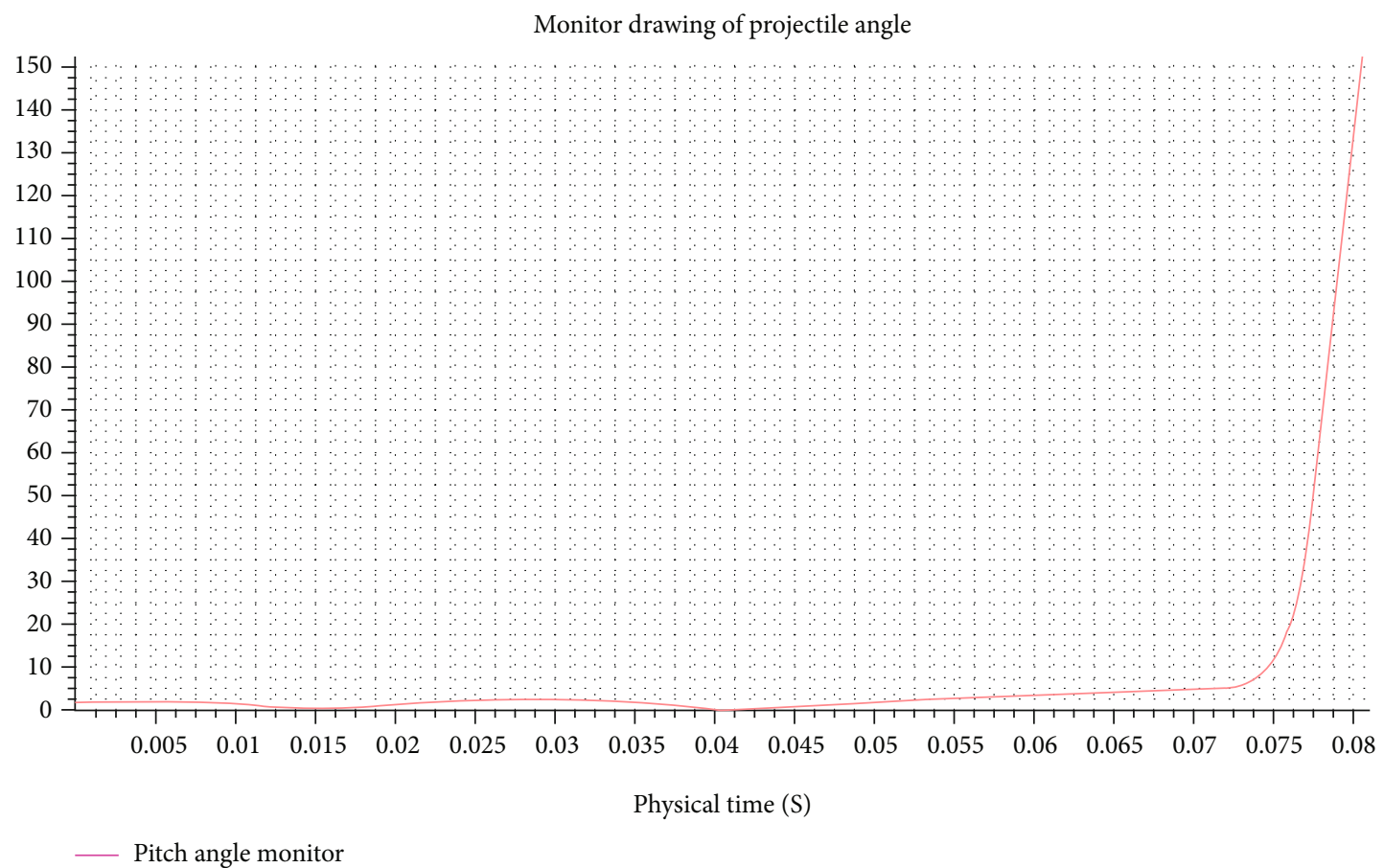

Figure 10: Graph of the elevation angle change of supercavitating projectile when centroid position equals $0.57 \mathrm{~L}$.

becomes relatively faster, and the number of tail shots increases.

\subsection{The Influence of the Position of the Center of Mass on the} Stability of Motion. With the analysis of the simulation results comparing the position of the center of mass $0.5 \mathrm{~L}$ and $0.6 \mathrm{~L}$ from the bullet head, the following issue can be drawn.
Tail shot motion is a way for underwater projectiles to maintain the stability, which satisfies that the moment the water exerts on the tail of the projectile is greater than or equal to the moment exerted by gravity, that is, the total moment $\sum M_{Y 1} \geq 0$. When $Q^{\bullet} \geq 0$, the force exerted by the water on the tail of the projectile is not enough to cause the projectile body to rotate in the opposite direction, and the projectile body will roll. There is a critical value $x_{\mathrm{cm}}$ 


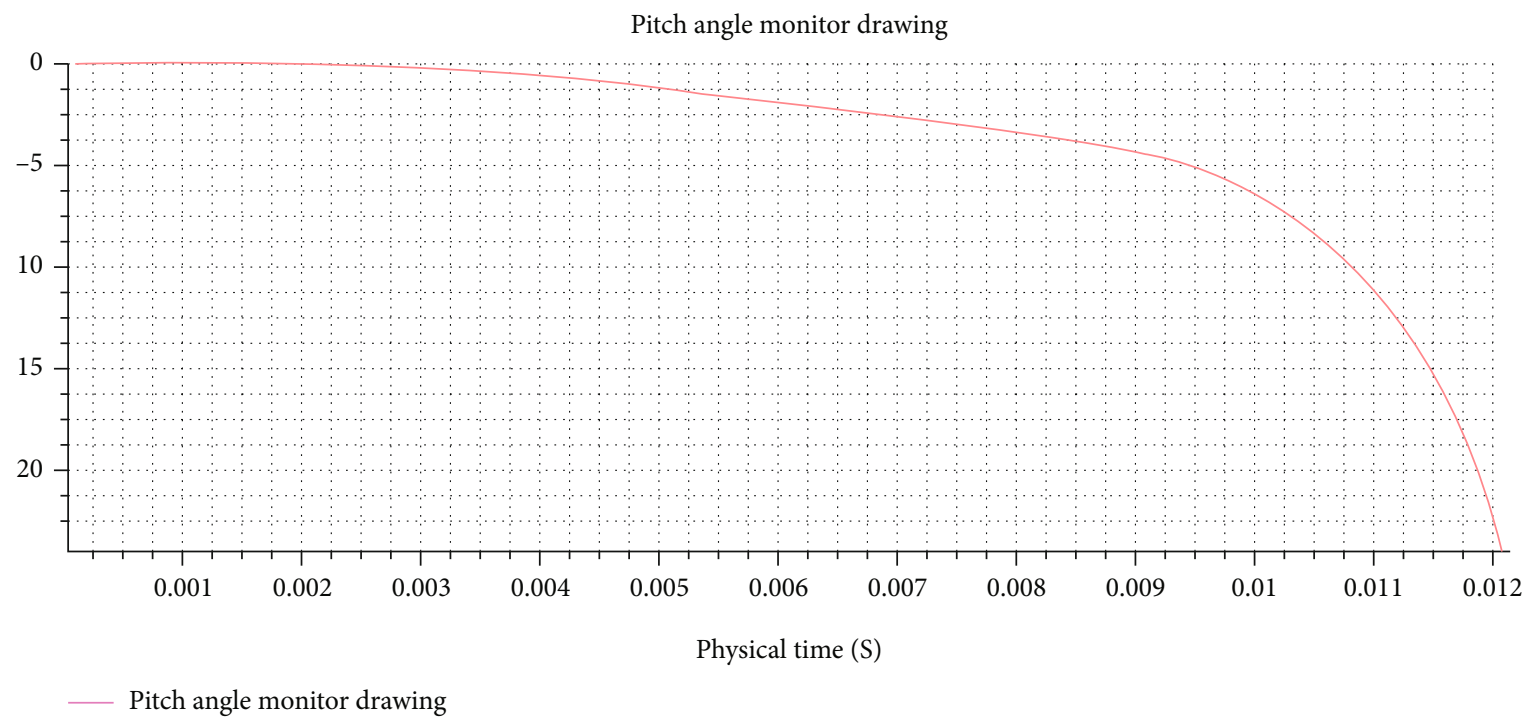

FIGURE 11: Graph of the elevation angle change of supercavitating projectile when centroid position equals $0.57 \mathrm{~L}$ and the projectile length equals $1.5 \mathrm{~L}$.

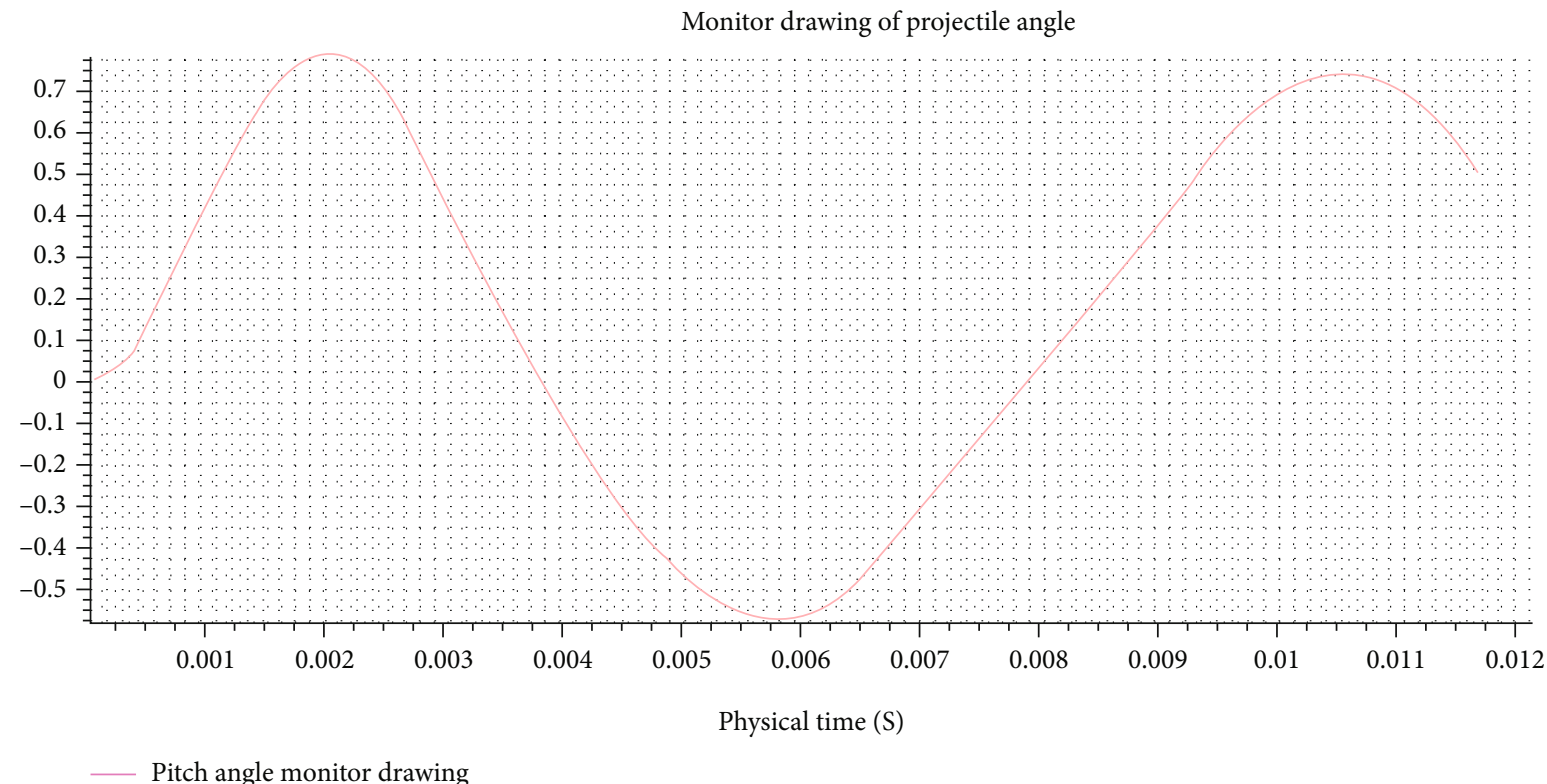

FIGURE 12: Graph of the elevation angle change of supercavitating projectile when centroid position equals $0.76 \mathrm{~L}$ and the projectile length equals $1.5 \mathrm{~L}$.

between $0.5 \mathrm{~L}$ and $0.6 \mathrm{~L}$. By changing the length of tungsten alloy and aluminum alloy, the position of the center of mass is brought forward to improve the motion stability.

3.4. Best Centroid Position. In order to further obtain the best centroid position, the next step of simulation is carried out, and three positions $0.55 \mathrm{~L}, 0.56 \mathrm{~L}$, and $0.57 \mathrm{~L}$ from the bullet head are chosen. The results are shown in Figures 7-10.

It can be seen from the above three simulation results that when the position of the center of mass is $0.55 \mathrm{~L}$ from the head of the projectile, the projectile is stable, while in the other two positions of the center of mass, the projectiles are all unstable. The three centers of mass positions are different by $0.1 \mathrm{~L}$. Therefore, the best center of mass position is $0.55 \mathrm{~L}$ from the bullet head, for this bullet shape.

In an underwater projectile of projectile length $L$, the distance from the center of the projectile to the head of the projectile is $0.63 \mathrm{~L}$, and the position of the critical center of mass is $0.08 \mathrm{~L}$ ahead of the center of the projectile. However, the conclusions of simulation research conducted on underwater projectiles are peculiar and not universal because this is only for the length of the projectile.

Two design schemes are proposed: (1) when the projectile length increases, the position of the critical center of mass is still $0.08 \mathrm{~L}$ ahead of the position of the centroid. (2) 
The distance between the critical center of mass and the centroid accounts for $8 \%$ of the projectile's length.

Increase the projectile length from $1 \mathrm{~L}$ to $1.5 \mathrm{~L}$, and keep the other shape parameters unchanged. The results can be found in Figures 11 and 12. Through calculation, the centroid position is $0.88 \mathrm{~L}$ away from the projectile head. Then, in the first design scheme, the critical centroid position should be set at $0.80 \mathrm{~L}$; in the second design scheme, the position of the critical center of mass should be set at $0.76 \mathrm{~L}$, and all other calculation conditions remain unchanged. Simulate the two positions of center of mass. From the simulation results of the projectile, it can be seen that when the center of mass is $0.76 \mathrm{~L}$ from the warhead, the underwater projectile is stable during its movement from $600 \mathrm{~m} / \mathrm{s}$ to $200 \mathrm{~m} / \mathrm{s}$. When the center of mass is $0.80 \mathrm{~L}$ from the warhead, the underwater projectile is stable. The movement process from $600 \mathrm{~m} / \mathrm{s}$ to $200 \mathrm{~m} / \mathrm{s}$ is unstable. It can be shown that the second design scheme is reasonable; that is, in the design process of underwater projectile, the distance from the center of mass to the centroid should be greater than or equal to $0.08 \mathrm{~L}$ of the projectile.

The influence of the position of the center of mass on the motion performance has been analyzed above. According to these analyses, an optimal position of the center of mass of the projectile can be obtained, and in the condition, a larger range is possible. Consider the projectile as a truncated cone. When the length of the tungsten alloy is exactly the distance from the center of mass to the head of the projectile, the center of mass is the most forward position. Set the length of the tungsten alloy at this time as $x$, the length of aluminum alloy as $0.1-x$, and the total mass as $m$.

$$
\frac{m}{2}<\frac{m+m 1}{2}<\frac{m 1}{18500-2700} \times 18500+\frac{m}{2}
$$

When the length of the tungsten alloy is the distance from the center of mass to the head of the projectile, no matter if the length of the tungsten alloy is increased or decreased, the position of the center of mass is far away from the head of the projectile.

\section{Conclusions}

In this paper, we study the motion stability and establish motion equations for supercavitating projectiles. Through theoretical analysis and simulation calculations, the optimal center of mass position is designed to optimize the motion performance of underwater supercavitating projectiles. The following conclusions can be drawn:

(1) When only changing the position of the center of mass, the center of mass close to the head of the projectile will reduce the energy consumption of a single tail shot movement, but the up and down vibration speed in the cavities of the projectile is relatively faster, and the number of tail shots increases. The energy consumption gap is nearly the same, which makes the range of no obvious difference; therefore, the center of mass is only increased by a centimeter order of range

(2) The position of the center of mass has a critical position; that is, when the value of the center of mass from the head of the projectile is less than this critical value, the underwater projectile can maintain the stability of motion. Otherwise, the result is the opposite

(3) The front part of the projectile body is made of tungsten alloy and the rear part of the projectile body is made of aluminum alloy. There is an optimal position of the center of mass, that is, the most forward position of the center of mass. When the distance from the center of mass to the head of the projectile equals to the length of the tungsten alloy, the position is the best center of mass position

The research in this paper can provide theoretical support for the optimal design of underwater supercavitating projectiles.

\section{Data Availability}

The data used to support the findings of this study are available from the corresponding author upon request.

\section{Conflicts of Interest}

The authors declare that there is no conflict of interest regarding the publication of this paper.

\section{References}

[1] Y. N. Savchenko, Control of supercavitation flow and stability of supercavitating motion of bodies/ /VKI special course on supercavitating flows, RTO2AVT and VKI, Brussels, 2001.

[2] H. Forouzani, B. Saranjam, and R. Kamali, "A study on the motion of high speed supercavitating projectiles," Journal of Applied Fluid Mechanics, vol. 11, no. 6, pp. 1727-1738, 2018.

[3] V. D. Kubenko and O. V. Gavrilenko, "Impact interaction of cylindrical body with a surface of cavity during supercavitation motion in compressible fluid," Journal of Fluids and Structures, vol. 25, no. 5, pp. 794-814, 2009.

[4] P. Wei, J. Hou, and T. F. Chen, "Analysis on the stability of supercavitation projectile," Applied Mechanics and Materials, vol. 157-158, pp. 58-61, 2012.

[5] W. Ping, H. Jian, C. Tingfeng, and L. Kuibin, Eds., "Analysis on the control of supercavitation projectile stability," in International Conference on Automatic Control and Artificial Intelligence (ACAI 2012), Xiamen, China, 2012.

[6] W. Ping and H. Jian, "Stability analysis and control of supercavitation projectile," in Proceedings of 2012 IEEE International Conference on Intelligent Control, Automatic Detection and High-End Equipment (ICADE 2012), pp. 80-83, Zhengzhou, China, 2012.

[7] R. Richard, P. Rudra, R. Deepak, C. Jeffery, and K. Ivan, "Impact dynamics of a supercavitating underwater projectile," in Proceedings of the 1997 ASME Design Engineering Technical Conferences, 16th Biennial Conference on Mechanical Vibration and Noise, Sacramento, CA, USA, September 1997. 
[8] S. S. Kulkarni and R. Pratap, "Studies on the dynamics of a supercavitating projectile," Applied Mathematical Modeling, vol. 24, no. 2, pp. 113-129, 2000.

[9] M. Ruzzene and F. Soranna, "Impact dynamics of elastic stiffened supercavitating underwater vehicles," Journal of Vibration and Control, vol. 10, no. 2, pp. 243-267, 2004.

[10] X.-T. Ding, S. J. Li, S.-J. Peng, and J. Wei, “Aerodynamic characteristics analysis and external trajectory simulation of highspeed cross-media water entry projectile," Journal of Physics: Conference Series, vol. 2079, no. 1, p. 012023, 2021.

[11] X. Zhao, X. Lyu, and D. Li, "Modeling of the tail slap for an underwater projectile within supercavitation," Mathematical Problems in Engineering, vol. 2019, 2019.

[12] P. Wei, J. Hou, and J. Wang, "Research on the influence of center of mass for stability of supercavition projectile," Ship Electronic Engineering, vol. 32, no. 1, pp. 97-98, 2012.

[13] X. S. Li and Y. F. Jiang, "Impact of gravity for stability of supervacuole projectile," Sichuan Ordnance Journal, vol. 32, no. 5, pp. 43-44, 2011.

[14] Q. C. Meng, Z. H. Zhang, G. JianNong, and J. B. Liu, "Analysis and calculation for tail-slaps of supercavitating projectiles," Combustion, Explosion, and Shock Waves, vol. 29, no. 1, pp. 56-60, 2009.

[15] D. X. Zhang and J. Hou, "Analysis of dynamic stability of supercavitating projectile," Sichuan Ordnance Journal., vol. 33, no. 9, pp. 21-22, 2012.

[16] C. G. Zhao, C. Wang, Y. J. Wei, and Q. P. Ma, “Analysis of the effect of mass center position on tailslap of supercavitating projectile," Journal of Beijing University of Aeronautics and Astronautics, vol. 40, no. 12, pp. 1754-1760, 2014.

[17] L. Zheng, Research on the Process of Projectile Entering Water across Medium, Harbin Engineering University, 2020.

[18] C. C. Qian, Numerical Simulation Study of Supercavitating Projectile Entering Water at High Speed, Nanjing University of Science and Technology, 2019.

[19] C. Huang, Research on Ballistic Characteristics of Transonic Supercavitating Projectile, Northwestern Polytechnical University, 2017.

[20] H. H. Shi, D. H. Zhou, Z. Dong, and H. X. Jia, "Research on flow and drag characteristics of under water successively fired supercavitating projectiles," Acta Aerodynamica Sinica, vol. 38, no. 4, pp. 771-779, 2020.

[21] W. J. Yi and T. H. Xiong, "Research on drag characteristics of natural supercavitation profile for high speed bodies," Ship Science and Technology, vol. 31, no. 1, pp. 38-42, 2009.

[22] J. S. Zhang, J. Z. Zhang, Y. J. Wei, and C. Wang, "Simulation analysis of impact loads on tails of supercavitating projectiles," Journal of Harbin Institute of Technology, vol. 43, no. 7, pp. 1922, 2011.

[23] M. Li, N. Liu, and Y. Y. Zou, Star-CCM+11.0, Machinery Industry Press, Beijing, 2017. 